\title{
Review of the clinical applications and technological advances of circulating tumor DNA in cancer monitoring
}

This article was published in the following Dove Press journal:

Therapeutics and Clinical Risk Management

II October 2017

Number of times this article has been viewed

\author{
Yi Changl,2,* \\ Bhairavi Tolani ${ }^{2, *}$ \\ Xiuhong Nie' \\ Xiuyi $\mathrm{Zhi}^{3}$ \\ $\mathrm{Mu} \mathrm{Hu}$ \\ Biao $\mathrm{He}^{2}$
}

'Department of Respiratory Medicine, Xuanwu Hospital, Capital Medical University, Beijing, China; ${ }^{2}$ Thoracic Oncology Program, Department of Surgery, Helen Diller Family Comprehensive Cancer Center, University of California, San Francisco, CA, USA; ${ }^{3}$ Department of Thoracic Surgery, Xuanwu Hospital, Capital Medical University, Beijing, China

*These authors contributed equally to this work
Correspondence: Bhairavi Tolani Thoracic Oncology Program, Department of Surgery, Helen Diller Family Comprehensive Cancer Center, University of California, San Francisco, 2340 Sutter St, N26I, San Francisco, CA $94 I I 5$, USA

Email bhairavi.tolani@ucsf.edu

\begin{abstract}
Circulating cell-free DNA (cfDNA) released by tumor cells, termed ctDNA, closely reflects the heterogeneity of primary cancers and their metastases. As a noninvasive, real-time monitoring biomarker, ctDNA is a promising tool for detecting driver gene mutations, assessing tumor burden and acquired resistance, and early diagnosis. However, isolation and enrichment of cfDNA is a big challenge due to the high degree of DNA fragmentation and its relatively low abundance in the bloodstream. This review aims to provide insights into the recent technological advances in acquisition of optimal quality cfDNA, the use of preservatives, isolation methods, processing timelines, and detection techniques. It also describes clinical applications of ctDNA in cancer patient management.
\end{abstract}

Keywords: circulating tumor DNA, ctDNA, liquid biopsy, cancer monitoring, tumor heterogeneity, targeted therapies, biomarkers

\section{Introduction}

Cancer is one of the most common causes of morbidity and mortality worldwide. The response rate, survival, and time to therapy failure have significantly improved with emergence of precision medicine, which requires knowledge of the specific molecular drivers in a tumor to select treatment interventions. Although tissue biopsies are considered to be the gold standard, they are still limited in their ability to identify genetic heterogeneity in a tumor or capture alterations that can occur as a result of selective pressures on tumor evolution. Additionally, biopsy carries some risks for patients and is also expensive and time-consuming. Circulating cell-free tumor DNA (ctDNA) released by tumor cells carries the molecular characteristics of primary tumors and can be collected chronologically for real-time monitoring of changes in tumor burden, including early diagnosis and assessment of treatment response and prognosis, thus mimicking a "liquid biopsy" concept. However, the application of ctDNA as a noninvasive monitoring tool in a clinical setting still faces several challenges. The biggest obstacle to surmount might be the detection limit: ctDNA may comprise $<0.1 \%$ of the total cell-free DNA (cfDNA), making detection of the tumor-specific fraction challenging and therefore requiring a highly sensitive technique. ${ }^{1}$ Furthermore, several factors (eg, type of matrix, storage conditions, or particular handling of blood samples) affect the ctDNA concentration and fragmentation values, and there is a lack of consensus regarding how to perform preanalytical and analytical procedures such as collection/ storage versus DNA sequence analyses. 
In this review, we summarize preanalytical considerations and recent technological advances in the acquisition of optimal quality cfDNA and also review studies focused on clinical applications of ctDNA in cancer patient management. We summarize recent data describing the clinical utility of ctDNA analyses for the most common tumor types and discuss the promise and challenges of its current and future clinical use.

\section{The biological characteristics of ctDNA}

cfDNA consists of nucleic acids released from necrotic and apoptotic cells into blood circulation and is thought to be derived from both healthy and cancer cells. ${ }^{2}$ Most cfDNA in healthy individuals is released from the bone marrow and white blood cells, whereas cfDNA in cancer patients (termed ctDNA) is derived from necrotic and apoptotic cancer cells. ${ }^{3}$ ctDNA contains heterogenetic defects (such as single-nucleotide mutations, methylation changes, and cancer-derived viral sequence elements) identical to the primary tumor and metastasis. The half-life of cfDNA is thought to range from 16 minutes to 2.5 hours, which enables cfDNA analysis to be considered as a dynamic monitoring read-out of disease burden, a characteristic that is particularly significant for monitoring cancer. ${ }^{4,5}$ cfDNA levels ( 0 to $>1,000 \mathrm{mg} / \mathrm{mL}$ ) in cancer patients are higher than those in healthy individuals $(0-100 \mathrm{ng} / \mathrm{mL})$, because as tumors increase in volume, so too does the level of ctDNA released from them. cfDNA is highly fragmented and usually consists of short segments ( $<185-200 \mathrm{bp}$ in length) that correspond to the DNA wrapped around a nucleosome plus a linker fragment; however, shorter ctDNA fragments have been reported in some tumor types (eg, hepatocellular carcinomas), as well as longer cfDNA fragments. ctDNA can be profiled in other body fluids (urine, cerebrospinal fluid [CSF], saliva) other than blood (plasma or serum), yet the exact nature and origin of cfDNA remains to be clarified. ${ }^{6,7}$ Variations in ctDNA concentration over time in the same individual can be several-fold (0.001\%-90\%) depending on factors such as tumor burden, stage, vascularity, cellular turnover, and response to therapy.,8 Because cfDNA is degraded by peripheral blood deoxyribonuclease (DNase) activity, the disproportion between DNase activity and ctDNA abundance leads to elevated ctDNA levels. ${ }^{9,10}$ Over time, the cfDNA level could increase due to the lysis of white blood cells and release of germline DNA, which could dilute ctDNA concentrations. Therefore, during molecular analysis, it is important to differentiate tumor-specific ctDNA from nontumor cfDNA.

\section{Approaches to ctDNA analysis Preanalytical considerations}

Since ctDNA carries the heterogenetic features of tumors, it has been thought of as a potential dynamic biomarker with diagnostic, predictive, and prognostic applications for various solid tumor types. However, isolation and enrichment of cfDNA is a great challenge given the high degree of cfDNA fragmentation and its low concentration in the bloodstream. ${ }^{11}$ Additionally, several sample handling factors affect the quality and quantity of ctDNA. Therefore, consistent and reproducible sampling factors described in the following section are essential to achieve superior quality results at the preanalytical stage.

\section{Serum versus plasma}

Several studies comparing cfDNA levels in paired plasma and serum samples have showed significantly higher cfDNA concentrations in serum. ${ }^{12,13}$ The elevated concentration of cfDNA in serum is due to the clotting process of white blood cells in the collection tube leading to their lysis. Consequently, cfDNA in serum is contaminated by germline DNA released from the lysed blood cells; thus, ctDNA is diluted by the presence of high concentrations of nonspecific tumor DNA. Although the use of plasma for the isolation and analysis of ctDNA has several advantages over serum, such as significantly higher cfDNA concentrations, it also has a few drawbacks which may decrease its utility. For example, red blood cell (RBC) lysis (hemolysis) can occur during improper specimen collection or mechanical processing of blood which causes rupturing of RBCs and release of their contents (including DNA) into the surrounding fluid. ${ }^{14-16}$ This surge in DNA contamination from nucleated cells can confound the analyses of ctDNA and render test results less accurate. While some studies support the use of serum over plasma, many reports state that cfDNA analyses from the plasma fraction of blood drawn is preferred over serum. ${ }^{17,18}$

\section{Preservative tube}

There are several types of preservative tubes used for blood collection. In most studies, standard EDTA collection tubes are commonly used for blood sampling. ${ }^{19}$ However, with the time delay between collection and analysis, the wildtype cfDNA concentration could increase due to lysis of hematological cells which release their own DNA, and thus reduce the relative percentage of tumor-specific ctDNA compared with the total amount of cfDNA. ${ }^{20}$ For practical reasons, it is often not possible to process and store blood samples after venipuncture. To address this problem, specialized cell-stabilizing blood collection tubes have been 
developed that not only reduce contamination by wild-type DNA but also maintain the quality and integrity of cfDNA for dependable downstream analysis. A study of the effect on the quality of cfDNA in 16 patients with metastatic cancer using different preservatives (EDTA, CellSave, and Streck's Cell-Free DNA BCT ${ }^{\circledR}$; Omaha, NE, USA) at different plasma isolation time points $(1,24$, and 96 hours) showed that both Streck's BCT and CellSave tubes preserve cfDNA/ctDNA quality well up to 96 hours and do not affect downstream cfDNA/ctDNA analyses by digital polymerase chain reaction (dPCR). ${ }^{21}$ In contrast, EDTA tubes processed 96 hours after blood withdrawal were not suitable for blood collection for subsequent cfDNA/ctDNA analysis because of the time-dependent increase in cfDNA concentration resulting from leukocyte lysis. Therefore, the recommended method is to isolate plasma from blood collected in either Streck's BCT or CellSave tubes. Another study reported that EDTA, Streck's BCT, and CellSave tubes were equivalent in stabilizing ctDNA for up to 6 hours before processing to isolate plasma. This may be due to the known inhibitory effect of EDTA on plasma DNase activity, but at 48 hours, the ctDNA level declined in the EDTA tube, whereas Streck's BCT and CellSave tubes more consistently stabilized ctDNA and wild-type DNA. ${ }^{22}$ Taken together, EDTA tubes could be a valid and cost-effective collection/storage method if the blood is processed within 6 hours, but if blood may have to be stored for longer periods of time ( $>6$ hours) before it is processed, Streck's BCT or CellSave tubes may be a more optimal option for blood collection. In addition to EDTA, plasma collection tubes can contain other anticoagulants such as heparin, which can influence the potential use of these samples for downstream applications. Avoiding heparincontaining tubes can improve the isolation of ctDNA as heparin can negatively impact sensitivity by inhibiting the PCR used for DNA amplification and quantification. ${ }^{23}$

Another important factor with respect to appropriate selection of a preservative tube is the storage temperature for these tubes, as variations in storage temperature can adversely affect genomic DNA concentrations. One study demonstrated that EDTA tubes were best processed within 2 hours or stored in a refrigerator $\left(2^{\circ} \mathrm{C}-8^{\circ} \mathrm{C}\right)$ until plasma separation, as storage at both room temperature $\left(22^{\circ} \mathrm{C}\right)$ and $37^{\circ} \mathrm{C}$ caused an increase in contaminating genomic DNA concentrations. ${ }^{11}$ This same study showed that Streck's BCT tubes could be stably stored at room temperature without major spikes in genomic DNA concentrations. ${ }^{11}$ These data show that certain blood collection tubes stabilize cfDNA levels and prevent the release of genomic DNA across various temperatures for a prolonged period of time. Thus, once a suitable tube is chosen, it is important to follow storage temperature guidelines based on the time needed to process blood samples.

\section{Extraction methods}

The development of high-throughput technologies for cfDNA isolation could improve the detection rate of cfDNA. A variety of automated systems could improve the reproducibility and robustness of the process. ${ }^{24-27}$ Conventional extraction methods, such as phenol-chloroform, alcohol precipitation, or a salting-out method, usually isolate higher concentrations than do DNA extraction kits. Nevertheless, these approaches are time-consuming and complex. cfDNA extraction may be carried out using affinity column-based, magnetic bead-based, polymer-based, and phenol-chloroform-based methods, or simply by filtration. Comparing DNA isolation methods such as columns and magnetic beads, one study reported that magnetic beads were a better choice. ${ }^{14}$ Some studies favored Qiagen's column-based QIAamp DNA Blood Mini Kit and report an $82 \%-92 \%$ recovery of cfDNA from serum while others advocated Macherey-Nagel's column-based NucleoSpin Plasma XS Kit in terms of yield, purity, and efficiency of small DNA fragment retrieval. ${ }^{14}$ However, in a comparative study, Roche's magnetic bead-based MagNA Pure produced 2-3 times more DNA than the MN columns and 5-10 times more than the Qiagen columns, thus making the use of magnetic beads more favorable. ${ }^{14,28}$ Different methods vary in their ability to recover particular fragment sizes of DNA, and these observations should be taken into account for cfDNA analysis in routine clinical practice.

\section{Optimal blood processing}

For cfDNA analysis, plasma or serum must be totally cellfree, so it is important that first centrifugation of the blood is done within a few hours of venipuncture to remove blood cells that may lyse and produce genomic DNA. The subsequent centrifugation should be performed to produce better cfDNA concentrations and improve purity. An optimized protocol for plasma isolation has been established whereby a blood sample is subjected to one centrifugation step at $1,200 \times g$ for 10 minutes, and a second one in which plasma is centrifuged at $16,000 \times g$ for 10 minutes..$^{19,29}$

\section{Influence of plasma storage conditions and freeze-thaw cycles}

Several studies attempted to define the optimal storage temperature before nucleic acid extraction and the effects of temperature variation on cfDNA stability. A study of the relationship between plasma storage conditions and cfDNA 
concentration showed that plasma sample storage for 2 weeks at $-80^{\circ} \mathrm{C}$ did not significantly affect plasma cfDNA levels and that the cfDNA integrity and quality decreased significantly after three freeze-thaw cycles. ${ }^{30}$

Due to the effect on cfDNA concentration and fragmentation, optimal preanalytical protocols should be developed. ${ }^{24,27,31}$ Current recommendations include the following: plasma is a better matrix than serum since it avoids contamination of cfDNA by genomic DNA from blood cells; blood must be processed within 6 hours after venipuncture when EDTA tubes are used, or within 96 hours when Streck's BCT or CellSave tubes are used; centrifugation ensures the absence of any cells in the plasma and a second high-speed centrifugation step is highly recommended; storage of plasma at $-80^{\circ} \mathrm{C}$; and repeated freeze-thaw cycles should be avoided. Although plasma storage at $-20^{\circ} \mathrm{C}$ for 4 weeks had no significant effect on DNA concentration, it negatively affected DNA quality, which was more fragmented. Thus, storage of plasma samples at $-80^{\circ} \mathrm{C}$ with minimum freeze-thaw cycles is recommended to preserve the quality of cfDNA. ${ }^{14,30}$

\section{Analytical methods for detecting ctDNA}

ctDNA detection analyses range in scale from single mutations to the entire genome. Current methodological approaches for the detection of ctDNA is categorized into two major types: 1) targeted methods to assay a few hot spot mutations with high sensitivity, and 2) untargeted methods with high costs, which allow simultaneous sequencing of millions of DNA fragments without prior sequence information and require the quantity of ctDNA to be around $5 \%-10 \%$ of the total DNA fraction.

The Sanger sequencing method for analyzing plasma ctDNA, developed in 1997, has many shortcomings, such as being low-throughput, time-consuming, expensive, and potential detection biases. ${ }^{32}$ Amplification refractory mutation system and other allele-specific approaches have been used to detect hot spot mutations (eg, KRAS, BRAF, and EGFR mutations) in serum and plasma with analytical sensitivity between $0.001 \%$ and $2 \%$, and some assays are available as kits that are approved for clinical use. ${ }^{33-39}$ Nevertheless, dPCR and digital droplet PCR assays are quantitative and highly sensitive, which can screen a sample to detect single target molecules and enrich mutant alleles. ${ }^{40-46}$ Therefore, these are generally suited to identifying hot spot mutations in cancers. ${ }^{47}$

In a clinical setting, investigations related to targeted therapies which probe clinically relevant genes and actionable alterations are preferred. Thus, Rosenfeld's group developed a method for tagged-amplicon deep sequencing as a personalized panel which can track a set of relevant genes associated with different tumor masses. ${ }^{48}$ To detect more complex genomic alterations such as chromosomal rearrangements, hybrid capture-based approaches (eg, cancer personalized profiling by deep sequencing [CAPP-Seq]) are used. ${ }^{49}$ By reducing the background error rate of sequencing via molecular barcoding strategies (Safe-Sequencing System, Safe-SeqS), these approaches can be used to identify ctDNA at allele fractions $<0.1 \% .{ }^{50}$ Even when ctDNA abundance is limited, the sensitivity of ctDNA detection can be further enhanced by using multiplexed patient-specific panels in conjunction with targeted sequencing approaches. ${ }^{51,52}$

When cfDNA is used as the starting material, ctDNA amplifications and deletions may be detected by whole exome sequencing (WES) and whole genome sequencing (WGS), which have the advantage that no previous sequence knowledge is necessary. ${ }^{51,53-57}$ Many driver genes are affected by copy number changes, and increased somatic copy number alterations (SCNAs) have been related to poor prognosis in several cancers. ${ }^{58-60}$ Such WGS has been applied to identify fetal aneuploidies, and it can also be used to detect cancer SCNAs. WGS and WES have a detection limit of 5\%-10\% for mutant alleles. ${ }^{61}$ It is feasible that in the near future, as costs decrease and new and improved analyses emerge, WES and WGS will be performed routinely for every diagnostic query as a generic test.

\section{Clinical applications of ctDNA and FDA-approved companion diagnostics}

Tissue biopsies are the gold standard diagnostic procedure for cancers. However, this procedure is invasive, painful, and carries some risks such as bleeding and damage to neighboring tissues, and is costly and time-consuming. Moreover, a tissue biopsy only samples one area of the tumor mass and fails to capture important biological information due to tumor heterogeneity. Some pancreatic tumors are difficult to access and therefore repeated sampling of tumors to monitor prognostic mutations is not practical. As a rapid and noninvasive means of dynamic monitoring and tumor prognosis, it has been verified that ctDNA reflects the molecular heterogeneity of a primary cancer and its metastases and is more tolerable to patients. Consequently, ctDNA analysis is considered to be very promising as a biomarker for early detection, identification of minimal residual disease (MRD), assessment of treatment response, and monitoring tumor evolution. 
The US FDA has approved a companion diagnostic, the $\operatorname{cobas}^{\circledR}$ EGFR Mutation Test v2, for two drugs, Tarceva ${ }^{\circledR}$ (erlotinib) and Tagrisso ${ }^{\mathrm{TM}}$ (osimertinib), used for the treatment of non-small-cell lung cancer (NSCLC). This FDAcleared real-time PCR test is used to detect mutations in the EGFR gene from plasma-derived ctDNA. Based on mutation status, this qualitative test aids in selecting NSCLC patients for treatment with targeted therapies such as erlotinib or osimertinib. ${ }^{62}$ Thus, several kits (in vitro diagnostics) designed for various cancer types can provide mutational profiling of ctDNA, which allows for the detection of the most common mutations and can guide treatment decisions for targeted treatment in these corresponding malignancies. Also, researchers at The University of Texas MD Anderson Cancer Center have reported another PCR-based liquidbiopsy test called Idylla ${ }^{\mathrm{TM}}$ BRAF Mutation Test which is useful for detecting BRAF ${ }^{\mathrm{V} 600}$ mutations. ${ }^{63}$ Thus, convenient and minimally invasive liquid biopsies have already reached the clinic and could enhance, if not replace, the more cumbersome and challenging tissue sampling. In addition, several companies, including Guardant Health, already provide liquid biopsies available on the market for detection of alterations in about 70 cancer genes from ctDNA. Furthermore, these lab-developed tests do not even require FDA approval to be sold on the market as long as the tests are performed by the original developer. Finally, ctDNA tests could be cheap alternatives to recruitment of patients for clinical trials testing investigational therapies against these mutations.

\section{Early diagnosis of cancer via ctDNA}

High mortality rates of various types of cancers make it imperative to find biomarkers for early detection of cancers before it is clinically obvious. However, the very low abundance of ctDNA in tumor samples and the fact that mutations present could be unknown make detection harder. Nevertheless, several studies have demonstrated the potential for noninvasive early diagnosis. Some of these studies have focused on quantifying cfDNA levels to distinguish benign from malignant disease. Two studies reported that cfDNA levels were eight and four times higher in patients with NSCLC than in control individuals. ${ }^{64}$ Two studies reported that a DNA cutoff level of $>20 \mathrm{mg} / \mathrm{mL}$ could discriminate between lung cancer patients and control subjects with a specificity of $83 \%$ and sensitivity of $79 \% .{ }^{64,65}$ In another study, ctDNA concentration was correlated with tumor volume. ${ }^{66}$ Furthermore, a meta-analysis of 10 studies regarding the diagnostic accuracy of ctDNA for lung cancer screening indicated a pooled specificity of $77 \%$ and a sensitivity of $80 \%$, concluding that
ctDNA alone is not sufficient for lung cancer screening. Similar studies have been performed in breast cancer, some of which found higher ctDNA levels in breast cancer patients than in control individuals, whereas other studies reported that there was no difference in ctDNA levels between the two groups. ${ }^{67}$ One factor that contributes to this current limitation in ctDNA detection is that copy numbers of ctDNA are generally very low compared with those of wild-type cfDNA. Another factor is the limited accuracy of the current sequencing methods in cancer. Several methods such as massively parallel sequencing or next-generation sequencing (NGS)based assays, and APCR and real-time PCR-based techniques are used for accurate cfDNA quantification which is both specific and sensitive. In particular, the beads, emulsification, amplification, and magnetics (BEAMing) system is thought to be the most specific and sensitive for cfDNA detection. This technique separates template molecules into individual reaction vessels by use of either microfluidics or into droplets in an oil emulsion. ${ }^{16}$ ctDNA ( $\left.<100 \mathrm{bp}\right)$ is typically shorter than the length of mononucleosomes ( $200 \mathrm{bp}$ long) and can be distinguished from other cfDNA by the presence of somatic mutations; techniques such as the BEAMing and NGS allow one to distinguish the proportion of ctDNA contained in cfDNA. Therefore, ctDNA quantification alone is not enough for detecting cancers.

The quality of ctDNA, including cfDNA integrity (ratio of shorter to longer fragments), telomeric cfDNA levels, and tumor-specific alterations (point mutations, SCNAs, rearrangements, promoter methylation), has been investigated in various cancers. These studies report that cfDNA integrity and telomeric cfDNA levels are lower in primary and metastatic breast cancer than in healthy controls. Chimonidou et al detected CDT6 promoter methylation, which is a tumor-specific mutation in plasma cfDNA in $13 \%-40 \%$ of breast cancer patients but not in healthy controls. ${ }^{68}$ Caceres et al screened 50 patients with ovarian cancer for hypermethylation status of BRCA1 and RAS-association domain family protein 1 tumor suppressor genes in serum or plasma, peritoneal fluid, and matched tumors. ${ }^{69}$ Because this study included patients with stage I disease, the results suggest that hypermethylation of particular genes is a common early event in ovarian tumorigenesis and may enhance the early detection of ovarian cancer based on noninvasive assays. There is good evidence from large validation studies on detection of ctDNA for diagnosis in gastrointestinal cancer that methylated SEPT9 and KRAS mutations in ctDNA are useful and accurate markers for colorectal cancer (CRC) diagnosis. ${ }^{70}$ In gastric carcinoma, current evidence suggests 
XAF1, HMLH1, RASSF1A, APC, and TIMP3 methylations are potential diagnostic markers. ${ }^{71}$ Methylated MINT2 appears to have both prognostic and diagnostic utility in gastric carcinoma, including detection of metastatic disease. ${ }^{71}$ KRAS mutations in ctDNA appear to be useful for diagnosis of pancreatic cancer. Available evidence suggests that concentration of overall ctDNA and TP53 249Ser mutations in ctDNA are important diagnostic markers of advanced-stage hepatitis B and aflatoxin-related hepatocellular carcinoma in African patients, but not in Caucasians. ${ }^{72}$ Recently, more emphasis has been placed on noncoding DNA, such as repetitive sequences like $A L U$ (which is a short interspersed nucleic element [SINE]) and long interspersed nucleotide elements such as LINE1. The integrity (size) of cfDNA $A L U$ sequences in blood has been reported to be a sensitive prognostic marker for the early stages of breast, testicular, prostate, nasopharyngeal, and ovarian cancer progression. ${ }^{73}$ However, more studies are needed to validate it as a clinically useful "universal" blood biomarker for multiple cancers. Tumor-specific loss of heterozygosity in cfDNA is also being investigated as an early detection marker, although a consensus is yet to be reached given the contradictory reports in the field. Also, circulating genomic DNA derived from tumors exists as nucleosomes, a histone octamer core wrapped twice by a 200 bp long DNA strand, and it can be quantified by an ELISA-based technique. Increased serum levels of nucleosomes concomitant with excessive deregulation of proteolytic activities caused by apoptotic cell death have been linked to breast cancer progression. ${ }^{73}$ Furthermore, viral cfDNA has been detected in the blood of patients whose cancers are associated with oncogenic viruses such as the human papillomavirus, hepatitis B virus, and Epstein-Barr virus (EBV). The presence of these viruses is linked to various malignancies, such as nasopharyngeal, cervical, head and neck, and hepatocellular cancer and lymphoma. The clinical use of EBV cfDNA from blood in the diagnosis and prognosis of nasopharyngeal carcinoma has been reported in a number of studies with large cohorts of patients. ${ }^{73}$ Taken together, several lines of evidence support the utility of ctDNA for early diagnosis in various cancers, but further large-scale validation studies are needed to corroborate future clinical applications.

Although these studies outline the possibility of ctDNA detection in early stage disease, one of the major problems is that because every cancer has a unique fingerprint, there is no universal marker that can be used for these diagnostic tests. Nevertheless, Illumina, a company well-known for DNA sequencing and genetic variation analysis, has invested in a new company called Grail. Grail aims to develop a test for early detection of cancer and to dramatically increase sensitivity in a cost-effective manner. By using NGS and combining sequencing depth and breadth of genomic coverage, Grail is performing a study to create a reference library of the cancer mutations in the blood for the most common cancers and also to catalog the background mutations found in matched healthy subjects as an early diagnostic and prognostic marker. ${ }^{74}$ This Blood Profiling Atlas Project hopes to offer early diagnosis of cancer via ctDNA. Another problem of screening in asymptomatic populations using ctDNA from the plasma is the risk of over-diagnosis and the introduction of false positives. Nowadays, a first step could involve the use of ctDNA for earlier diagnosis of disease in symptomatic patients, who then may undergo a dynamic monitoring procedure.

\section{ctDNA as a prognostic marker}

Baseline ctDNA levels in cancer patients as a new prognostic and predictive tool has been extensively studied and tested. ${ }^{65}$ Further, while several studies report that high levels of ctDNA result in shorter overall survival (OS) of patients, other contradictory reports state that increased levels of ctDNA are not associated with OS or progression-free survival (PFS). ${ }^{75,76}$ These inconsistent results indicate that ctDNA levels have a limited prognostic value. In contrast, quantification of tumor-specific mutations in ctDNA seems to be more relevant. It is well known that the PIK3CA gene can be found in $40 \%$ of all breast tumors, making these mutations suitable biomarkers. ${ }^{47,77,78}$ A study in a 313 stage I-III breast cancer patients cohort reported that in patients with high levels of PIK3CA mutant ctDNA, PFS and OS rates were significantly shorter than in patients with low or no detectable amount of ctDNA ${ }^{47}$ In ovarian cancer, promoter methylation of MLH1 was found to be increased at relapse and methylation was acquired after chemotherapy in $25 \%$ of samples. ${ }^{79}$ This aberrant methylation predicts poor OS of patients, independent of time to progression and age. ${ }^{79}$ In prostate cancer patients, androgen receptor (AR) antagonist gene aberrations are established resistance markers and can be detected from plasma, and some studies found that several AR gene aberrations and amplifications detected in plasma correlated with worse PFS. ${ }^{80}$ Several studies identified ctDNA as a predictor of clinical outcome in melanoma patients, reporting that lower concentrations of basal mutant BRAF ctDNA correlated with a higher response rate and longer PFS for patients treated with immunotherapies, and that monitoring ctDNA levels can provide a "snapshot" of disease progression. Moreover, the simultaneous determination of three biomarkers (total cfDNA, integrity, 
and methylated RASSF1A) could improve the diagnostic performance in melanoma. ${ }^{81,82}$ NSCLC patients with elevated circulating EGFR copy number levels had a lower OS and PFS compared with patients harboring low EGFR copy number levels in plasma. ${ }^{83}$ Furthermore, patients with high levels of EGFR-activating mutations in tyrosine kinase inhibitor (TKI)-naïve plasma samples have longer OS and PFS. ${ }^{83}$ Apropos the prognostic value of KRAS mutation levels in lung cancer patients, some studies have shown that patients with detectable KRAS mutations have a significantly shorter OS and/or PFS compared to patients with wild-type KRAS. ${ }^{84}$ In summary, prognostic information offered by ctDNA mutation detection could facilitate early recognition of metastatic disease and personalize treatment algorithms to maximize outcomes, but a reliable cutoff remains to be determined.

\section{Monitoring treatment efficiency}

Detection of ctDNA is well-suited for real-time monitoring of cancer burden in response to therapy because of its short halflife as well as the ease and reduced risk of repetitive liquid biopsies relative to imaging or tissue biopsies. A number of studies have employed ctDNAs as biomarkers of metastatic disease activities to monitor disease response and overall disease burden. A variety of studies found decreased levels of ctDNA after surgery and/or chemotherapy. In one study, an increase in ctDNA correlated with disease progression in $17 / 19$ metastatic breast cancer patients, and reported at an average of 5 months before progression was confirmed with imaging. ${ }^{85}$ Therefore, ctDNA seemed to be better and more sensitive for monitoring tumor burden than CA153 and circulating tumor cells. Several studies have shown that ctDNA can be a surrogate for tumor burden in patients with NSCLC. One particular study reported that levels of ctDNA could be significantly correlated with tumor volume, differentiated between residual disease and treatment-related imaging changes, and furnished earlier response assessments than radiographic approaches. ${ }^{66}$ Another study showed that in patients with blood-based EGFR mutation-positive results at baseline, the dynamic change in EGFR status in blood samples was associated with efficacy outcomes. ${ }^{86}$ Another examined the correlation between ctDNA and total tumor burden defined by positron emission tomography parameters in advanced NSCLC, finding no correlation between ctDNA, metabolic tumor volume, and total lesion glycolysis. ${ }^{87}$ The results suggested that cfDNA is not simply a direct reflection of tumor burden and is more complex than what was originally thought. These incongruities could be attributed to different methods utilized for extraction and quantification. ${ }^{87}$ Similar conclusions have been reached with other cancer types including metastatic melanoma undergoing immune checkpoint blockade, metastatic colorectal cancer, and primary gynecological malignancies ${ }^{87-89}$ All these studies reported that ctDNA analysis cannot completely replace imaging, but can be used in conjunction with imaging to gain additional insight into tumor response.

\section{Monitoring of MRD and early detection of recurrence}

Mutation tracking also can be a significant predicator of early relapse and MRD. Currently, effective methods to identify MRD in patients who are cured by surgery or adjuvant chemotherapy are still lacking. The tumor-node-metastasis staging system stratifies patients by risk for recurrence but does not identify whether residual tumors are present after surgical resection. Tumor metastases will carry many of the mutations that were present in the primary tumor, although they are usually not genetically identical to the primary tumor. This presents the possibility of detecting these mutations in ctDNA as an early indicator of recurrence and a potential marker of residual disease.

Monitoring ctDNA for residual disease has been used in patients with breast cancer, melanoma, colorectal cancer, and lung cancer. ${ }^{90-95}$ In NSCLC, when the sensitivity and specificity of CAPP-Seq for MRD detection was assessed using plasma samples from NSCLC patients, ctDNA was detected in $100 \%$ of patients with stage II-IV NSCLC and in $50 \%$ of patients with stage $\mathrm{I} .{ }^{66}$ The best example has been in a cohort of patients with colorectal cancer undergoing resection with curative intent. ${ }^{37}$ In that study, all patients with measurable postoperative levels of ctDNA encountered relapse, whereas all patients with imperceptible postoperative levels of ctDNA remained disease free. Tumor recurrence after resection has been associated with both KRAS mutations and hypermethylation of p16 in ctDNA. ${ }^{84}$ In one study, the 2-year recurrence-free survival after curative resection was $66 \%$ in patients with detectable ctDNA levels compared with $100 \%$ in those without detectable ctDNA. ${ }^{96}$ In a study of ctDNA for early detection of metastasis in women with primary breast cancer who were treated with surgery only with a curative intent, before metastasis was detected clinically, ctDNA was detected in $86 \%$ of patients with an average lead time of 11 months. ${ }^{97}$

\section{Identification of resistance mutations}

It is crucial to detect the appearance of resistance to chemotherapy and targeted agents to avoid continuing ineffective therapies in the management of cancers. Studies of ctDNA 
have provided new insights into this area. In breast cancer, PIK3CA hot spot mutations are one of the actionable targets for anastrazole. An ESR1 mutation predicts the resistance to endocrine therapy. Detection of ctDNA is widely used in NSCLC, including the identification of actionable mutations for targeted treatments (EGFR, ALK, ROS1, RET rearrangements, HER2 insertions, and MET) and monitoring acquired resistance mutations (T790M mutation, amplification of the MET receptor tyrosine kinase or ERBB2, and mutations in PIK3CA, BRAF, STAT3, or AXL amplifications). ${ }^{52}$ The most commonly known TKI targets in NSCLC are EGFRactivating mutations, but acquired resistance is the bottleneck for first-generation and second-generation EGFR TKIs and is frequently due to secondary EGFR T790M mutations, which are present in $\sim 50 \%-60 \%$ of resistant cases. ${ }^{98,99}$ A study in 503 CRC patients confirmed the utility of detecting KRAS, BRAF, and PIK3CA mutations in ctDNA in CRC patients on regorafenib therapy and demonstrated that mutations present in ctDNA change dynamically during chemotherapy treatment. ${ }^{100}$ Therefore, ctDNA detection can be used to illustrate the mechanisms of tumor resistance and guide an earlier selection of appropriate therapies that may improve clinical outcome.

\section{Examples of clinical application of ctDNA in monitoring cancer progression}

The analysis of ctDNA is expected to be a way to monitor the status of cancer in real time. Several case reports in a variety of cancers, including sarcoma, metastatic malignant melanoma, rectal cancer, prostate cancer, breast cancer and lung cancer, have focused on the relationship between the changes of ctDNA levels, tumor burden, and metastatic activity in cancer patients. ${ }^{100-103}$ In the following section, we present some case reports that describe the factors that influence ctDNA analyses. ${ }^{104,105}$

\section{Breast cancer}

A 45-year-old woman with metastatic breast cancer was treated with bevacizumab and paclitaxel. ${ }^{106}$ The lung metastases decreased but carcinomatous meningitis developed rapidly and she died. While the patient was being treated with bevacizumab and paclitaxel, NGS analysis was performed on ctDNA isolated from peripheral blood samples collected serially (at 0 as pretreatment and 30,60, 120, 150, 180, and 210 days after the first treatment). A seven-nucleotide deletion of TP53 mutation was detected and this tumor-specific mutation was identified in secondary metastases. ${ }^{107}$ Results showed that the value of the allelic fraction (AF) of the TP53 mutation dropped, corresponding to the effect of treatment before the change in carcinoembryonic antigen (CEA), a standard biomarker for disease management of colorectal cancer. Contributing factors may be the short half-life time ( $\sim 2$ hours) of ctDNA, which allow us to observe changes in the timescale of hours. Therefore, detection of plasma ctDNA after several days provided important information on the therapeutic effect. The ctDNA levels suddenly increased 2 months before the CEA. This sudden upregulation in ctDNA release resulted due to dynamic alterations in the metastatic lesion, and the following ctDNA measurement was consistently recorded at $2-4 \times 10^{-4}$ irrespective of disease progression and increase in CEA value. This phenomenon might be caused by the elevation of total ctDNA comprising other mutations that affect the decreased value of a TP53 mutation. This case shows that ctDNA can detect rapid changes in tumor burden more sensitively than the protein markers CEA or CA153. However, further investigations are needed to confirm the significance of these highly sensitive changes.

\section{NSCLC}

A case of long-term monitoring of a 57-year-old female NSCLC patient for analysis of EGFR mutations (L858R and T790M) using ctDNA illustrates that the clinical evolution of the patient and the changes in EGFR mutations in ctDNA isolated from serum/plasma or CSF correlate well. ${ }^{100}$ This case report shows that analysis of EGFR mutations can detect an early progression of the disease in asymptomatic patients and that CSF can be used as an alternative "liquid biopsy source," with applications for the management of brain tumors and metastases. A case report showing that ctDNA detected the EML4-ALK fusion in a patient with EGFR-mutated NSCLC and that adding ALK inhibitors was successful proved that it is feasible to identify the resistance mechanism of targeted treatment using ctDNA detection. ${ }^{100}$

\section{A pilot ctDNA detection experiment in 168 patients with diverse cancers}

A study of NGS on plasma-derived ctDNA in 168 patients with diverse cancers (54 cancer-related gene panel including amplifications in ERBB2, EGFR, and MET) found that 58\% of patients had a molecular alteration and that in $71 \%$ of the patients with alterations, there was an alteration that was potentially pharmacologically tractable by an FDA-approved drug. ${ }^{108}$ In that study, $28.6 \%$ (16/56) of patients with brain tumors had a molecular alteration in their ctDNA test, suggesting that liquid biopsies may supplement the assessment 
of this difficult-to-biopsy site. The degree to which tissue biopsies and ctDNA molecular results were concordant was related to the time interval between the acquisitions of the tissue versus blood samples. Taken together, these data suggest that ctDNA tests may have clinical utility that merits additional investigation. ${ }^{108}$

\section{Conclusion and perspective}

The detection and analysis of ctDNA has emerged as a potential blood-based "liquid biopsy" for early diagnosis, assessment of treatment response, and prognosis in cancer patients. However, challenges remain that should be addressed so that detection of plasma ctDNA may eventually be implemented into routine clinical practice. Although many studies have focused on the clinical applications of ctDNA, data regarding the actual origin, kinetics, and mechanisms of ctDNA release and clearance are limited and often contradictory. Therefore, the biology of cfDNA remains unclear. A better understanding of the origin and biology of ctDNA and cfDNA would aid the implementation of liquid biopsies. It is also not clear whether ctDNA could represent all relevant metastatic cell clones or whether ctDNA could represent DNA from distinct subclones that can induce disease progression and/or acquired resistance. Further clinical evaluations, comparative sequence analyses of plasma DNA, and biopsies in combination with imaging studies and detailed functional studies are needed to assess the disease.

The discrepancies amongst several studies reported to date including the relatively low ctDNA sensitivity in detection could, perhaps, be attributed to the lack of consensus in the selection of technical approach, sample type (serum vs plasma), storage conditions, candidate mutation detected, or appropriate detection methods. Therefore, regarding the high degree of fragmentation of cfDNA and its low concentration in the bloodstream, a consensus of preanalytical and analytical techniques needs to be developed. Although many advanced technologies for identifying ctDNA in circulation have been improved, the major hurdle for clinical use is probably the fact that most methods are not applicable in clinical practice given the time, costs, and technical equipment required. ctDNA can be used as a noninvasive cancer biomarker and may serve as an alternative or supplemental material for driver gene mutation analysis in clinical practice for cancer patients unable to provide tissue-based samples. Additionally, ctDNA can play complementary roles in diagnosis and serve as prognostic or predictive biomarker in patients with cancers. With the development of more sensitive and specific methods, assays of ctDNA might replace invasive procedures and may be used to perform genomic analysis in the near future.

\section{Disclosure}

The authors report no conflicts of interest in this work.

\section{References}

1. Zhang W, Xia W, Lv Z, Ni C, Xin Y, Yang L. Liquid biopsy for cancer: circulating tumor cells, circulating free DNA or exosomes? Cell Physiol Biochem. 2017;41(2):755-768.

2. Thierry AR, El Messaoudi S, Gahan PB, Anker P, Stroun M. Origins, structures, and functions of circulating DNA in oncology. Cancer Metastasis Rev. 2016;35(3):347-376.

3. Leon SA, Shapiro B, Sklaroff DM, Yaros MJ. Free DNA in the serum of cancer patients and the effect of therapy. Cancer Res. 1977;37(3): 646-650.

4. Chen XQ, Stroun M, Magnenat JL, et al. Microsatellite alterations in plasma DNA of small cell lung cancer patients. Nat Med. 1996;2(9): 1033-1035.

5. Chen X, Bonnefoi H, Diebold-Berger S, et al. Detecting tumor-related alterations in plasma or serum DNA of patients diagnosed with breast cancer. Clin Cancer Res. 1999;5(9):2297-2303.

6. Heitzer E, Ulz P, Geigl JB. Circulating tumor DNA as a liquid biopsy for cancer. Clin Chem. 2015;61(1):112-123.

7. Bettegowda C, Sausen M, Leary RJ, et al. Detection of circulating tumor DNA in early- and late-stage human malignancies. Sci Transl Med. 2014;6(224):224ra24.

8. Wang J, Bettegowda C. Applications of DNA-based liquid biopsy for central nervous system neoplasms. J Mol Diagn. 2017;19(1):24-34.

9. Cox RA, Gokcen M. Comparison of serum DNA, native DNA-binding and deoxyribonuclease levels in ten animal species and man. Life Sci. 1976;19(10):1609-1614.

10. Tamkovich SN, Cherepanova AV, Kolesnikova EV, et al. Circulating DNA and DNase activity in human blood. Ann N Y Acad Sci. 2006; 1075:191-196.

11. Norton SE, Luna KK, Lechner JM, Qin J, Fernando MR. A new blood collection device minimizes cellular dna release during sample storage and shipping when compared to a standard device. J Clin Lab Anal. 2013;27(4):305-311.

12. Lee TH, Montalvo L, Chrebtow V, Busch MP. Quantitation of genomic DNA in plasma and serum samples: higher concentrations of genomic DNA found in serum than in plasma. Transfusion. 2001;41(2): 276-282.

13. Jung M, Klotzek S, Lewandowski M, Fleischhacker M, Jung K. Changes in concentration of DNA in serum and plasma during storage of blood samples. Clin Chem. 2003;49(6 Pt 1):1028-1029.

14. Bronkhorst AJ, Aucamp J, Pretorius PJ. Cell-free DNA: preanalytical variables. Clin Chim Acta. 2015;450:243-253.

15. Ko DH, Won D, Jeong TD, et al. Comparison of red blood cell hemolysis using plasma and serum separation tubes for outpatient specimens. Ann Lab Med. 2015;35(2):194-197.

16. Volik S, Alcaide M, Morin RD, et al. Cell-free DNA (cfDNA): clinical significance and utility in cancer shaped by emerging technologies. Mol Cancer Res. 2016;14(10):898-908.

17. Umetani N, Hiramatsu S, Hoon DS. Higher amount of free circulating DNA in serum than in plasma is not mainly caused by contaminated extraneous DNA during separation. Ann N Y Acad Sci. 2006; 1075:299-307.

18. Vallée A, Marcq M, Bizieux A, Kouri CE. et al. Plasma is a better source of tumor-derived circulating cell-free DNA than serum for the detection of EGFR alterations in lung tumor patients. Lung Cancer. 2013;82(2):373-374.

19. El Messaoudi S, Rolet F, Mouliere F, Thierry AR. Circulating cell free DNA: preanalytical considerations. Clin Chim Acta. 2013;424: 222-230. 
20. Norton SE, Lechner JM, Williams T, Fernando MR. A stabilizing reagent prevents cell-free DNA contamination by cellular DNA in plasma during blood sample storage and shipping as determined by digital PCR. Clin Biochem. 2013;46:1561-1565.

21. van Dessel LF, Beije N, Helmijr JC, et al. Application of circulating tumor DNA in prospective clinical oncology trials - standardization of preanalytical conditions. Mol Oncol. 2017;11(3):295-304.

22. Kang Q, Henry NL, Paoletti C, et al. Comparative analysis of circulating tumor DNA stability in K3EDTA, Streck, and CellSave blood collection tubes. Clin Biochem. 2016;49(18):1354-1360.

23. Yokota M, Tatsumi N, Nathalang O, et al. Effects of heparin on polymerase chain reaction for blood white cells. J Clin Lab Anal. 1999; 13(3):133-140.

24. Devonshire AS, Whale AS, Gutteridge A, et al. Towards standardization of cell-free DNA measurement in plasma: controls for extraction efficiency, fragment size bias and quantification. Anal Bioanal Chem. 2014;406(26):6499-6512.

25. Fong SL, Zhang JT, Lim CK, Eu KW, Liu Y. Comparison of 7 methods for extracting cell-free DNA from serum samples of colorectal cancer patients. Clin Chem. 2009;55(3):587-589.

26. Malentacchi F, Pizzamiglio S, Verderio P, et al. Influence of storage conditions and extraction methods on the quantity and quality of circulating cell-free DNA (ccfDNA): the SPIDIA-DNAplas external quality assessment experience. Clin Chem Lab Med. 2015;53(12):1935-1942.

27. Mauger F, Dulary C, Daviaud C, Deleuze JF, Tost J. Comprehensive evaluation of methods to isolate, quantify, and characterize circulating cell-free DNA from small volumes of plasma. Anal Bioanal Chem. 2015; 407(22):6873-6878

28. Jorgez CJ, Dang DD, Simpson JL, et al. Quantity versus quality: optimal methods for cell-free DNA isolation from plasma of pregnant women. Genet Med. 2006;8(10):615-619.

29. El Messaoudi S, Mouliere F, Du Manoir S, et al. Circulating DNA as a strong multimarker prognostic tool for metastatic colorectal cancer patient management care. Clin Cancer Res. 2016;22(12):3067-3077.

30. Chan KC, Yeung SW, Lui WB, Rainer TH, Lo YM. Effects of preanalytical factors on the molecular size of cell-free DNA in blood. Clin Chem. 2005;51(4):781-784.

31. Goldshtein H, Hausmann MJ, Douvdevani A. A rapid direct fluorescent assay for cell-free DNA quantification in biological fluids. Ann Clin Biochem. 2009;46(Pt 6):488-494.

32. Vendrell JA, Mau-Them FT, Béganton B, Godreuil S, Coopman P, Solassol J. Circulating cell free tumor DNA detection as a routine tool for lung cancer patient management. Int J Mol Sci. 2017;18(2):264.

33. Mouliere F, El Messaoudi S, Gongora C, et al. Circulating cell-free DNA from colorectal cancer patients may reveal high KRAS or BRAF mutation load. Transl Oncol. 2013;6(3):319-328.

34. Mouliere F, El Messaoudi S, Pang D, Dritschilo A, Thierry AR. Multimarker analysis of circulating cell-free DNA toward personalized medicine for colorectal cancer. Mol Oncol. 2014;8(5):927-941.

35. Newton CR, Graham A, Heptinstall LE, et al. Analysis of any point mutation in DNA. The amplification refractory mutation system (ARMS). Nucleic Acids Res. 1989;17(7):2503-2516.

36. Spindler KL, Pallisgaard N, Vogelius I, Jakobsen A. Quantitative cell-free DNA, KRAS, and BRAF mutations in plasma from patients with metastatic colorectal cancer during treatment with cetuximab and irinotecan. Clin Cancer Res. 2012;18(4):1177-1185.

37. Douillard JY, Ostoros G, Cobo M, et al. Gefitinib treatment in EGFR mutated Caucasian NSCLC circulating-free tumor DNA as a surrogate for determination of EGFR status. J Thorac Oncol. 2014;9(9): $1345-1353$

38. Aung KL, Donald E, Ellison G, et al. Analytical validation of BRAF mutation testing from circulating free DNA using the amplification refractory mutation testing system. J Mol Diagn. 2014;16(3): 343-349.

39. Schreuer M, Meersseman G, van Den Herrewegen S, et al. Applications for quantitative measurement of BRAF V600 mutant cell-free tumor DNA in the plasma of patients with metastatic melanoma. Melanoma Res. 2016;26(2):157-163.
40. Taly V, Pekin D, Benhaim L, et al. Multiplex picodroplet digital PCR to detect KRAS mutations in circulating DNA from the plasma of colorectal cancer patients. Clin Chem. 2013;59(12):1722-1731.

41. Oxnard GR, Paweletz CP, Kuang Y, et al. Noninvasive detection of response and resistance in EGFR-mutant lung cancer using quantitative next-generation genotyping of cell-free plasma DNA. Clin Cancer Res. 2014;20(6):1698-1705.

42. Toro PV, Erlanger B, Beaver JA, et al. Comparison of cell stabilizing blood collection tubes for circulating plasma tumor DNA. Clin Biochem. 2015;48(15):993-998.

43. Baker M. Digital PCR hits its stride. Nat Methods. 2012;9(6): 541-544.

44. Day E, Dear PH, McCaughan F. Digital PCR strategies in the development and analysis of molecular biomarkers for personalized medicine. Methods. 2013;59(1):101-107.

45. Chang GA, Tadepalli JS, Shao Y, et al. Sensitivity of plasma BRAF mutant and NRAS mutant cell-free DNA assays to detect metastatic melanoma in patients with low RECIST scores and non-RECIST disease progression. Mol Oncol. 2016;10(1):157-165.

46. Shoda K, Ichikawa D, Fujita Y, et al. Monitoring the HER2 copy number status in circulating tumor DNA by droplet digital PCR in patients with gastric cancer. Gastric Cancer. 2017;20(1):126-135.

47. Oshiro C, Kagara N, Naoi Y, et al. PIK3CA mutations in serum DNA are predictive of recurrence in primary breast cancer patients. Breast Cancer Res Treat. 2015;150(2):299-307.

48. Gale D, Plagnol V, Lawson A, et al. Analytical performance and validation of an enhanced TAm-Seq circulating tumor DNA sequencing assay. AACR. 2016;76: abstract 3639.

49. Bratman SV, Newman AM, Alizadeh AA, Diehn M. Potential clinical utility of ultrasensitive circulating tumor DNA detection with CAPPSeq. Expert Rev Mol Diagn. 2015;15(6):715-719.

50. Schmitt MW, Kennedy SR, Salk JJ, Fox EJ, Hiatt JB, Loeb LA. Detection of ultra-rare mutations by next-generation sequencing. Proc Natl Acad Sci U S A. 2012;109(36):14508-14513.

51. Forshew T, Murtaza M, Parkinson C, et al. Noninvasive identification and monitoring of cancer mutations by targeted deep sequencing of plasma DNA. Sci Transl Med. 2012;4(136):136ra68.

52. Newman AM, Lovejoy AF, Klass DM, et al. Integrated digital error suppression for improved detection of circulating tumor DNA. Nat Biotechnol. 2016;34(5):547-555.

53. Chan KC, Jiang P, Chan CW, et al. Noninvasive detection of cancerassociated genome-wide hypomethylation and copy number aberrations by plasma DNA bisulfite sequencing. Proc Natl Acad Sci U S A. 2013; 110(47):18761-18768.

54. Lo YM, Chan KC, Sun H, et al. Maternal plasma DNA sequencing reveals the genome-wide genetic and mutational profile of the fetus. Sci Transl Med. 2010;2(61):61ra91.

55. Murtaza M, Dawson SJ, Tsui DW, et al. Non-invasive analysis of acquired resistance to cancer therapy by sequencing of plasma DNA. Nature. 2013;497(7447):108-112.

56. Jamal-Hanjani M, Wilson GA, Horswell S, et al. Detection of ubiquitous and heterogeneous mutations in cell-free DNA from patients with earlystage non-small-cell lung cancer. Ann Oncol. 2016;27(5):862-867.

57. Pereira E, Camacho-Vanegas O, Anand S, et al. Personalized circulating tumor DNA biomarkers dynamically predict treatment response and survival in gynecologic cancers. PLoS One. 2015;10(s12):e0145754.

58. Carter SL, Eklund AC, Kohane IS, Harris LN, Szallasi Z. A signature of chromosomal instability inferred from gene expression profiles predicts clinical outcome in multiple human cancers. Nat Genet. 2006;38(9): 1043-1048.

59. Zack TI, Schumacher SE, Carter SL, et al. Pan-cancer patterns of somatic copy number alteration. Nat Genet. 2013;45(10):1134-1140.

60. Heitzer E, Ulz P, Geigl JB, Speicher MR. Non-invasive detection of genome-wide somatic copy number alterations by liquid biopsies. Mol Oncol. 2016;10(3):494-502.

61. Heitzer E, Ulz P, Belic J, Gutschi S, et al. Tumor-associated copy number changes in the circulation of patients with prostate cancer identified through whole-genome sequencing. Genome Med. 2013;5(4):30. 
62. Kwapisz D. The first liquid biopsy test approved. Is it a new era of mutation testing for non-small cell lung cancer? Ann Transl Med. 2017;5(3):46.

63. Janku F, Huang HJ, Claes B, et al. BRAF mutation testing in cell-free DNA from patients with advanced cancers using a rapid, automated molecular diagnositics system. Mol Cancer Ther. 2016;15(6):1397-1404.

64. Sozzi G, Conte D, Leon M, et al. Quantification of free circulating DNA as a diagnostic marker in lung cancer. J Clin Oncol. 2003;21(21): 3902-3908.

65. Catarino R, Coelho A, Araújo A, et al. Circulating DNA: diagnostic tool and predictive marker for overall survival of NSCLC patients. PLoS One. 2012;7(6):e38559.

66. Newman AM, Bratman SV, To J, et al. An ultrasensitive method for quantitating circulating tumor DNA with broad patient coverage Nat Med. 2014;20(5):548-554.

67. Nicolini C, Ens C, Cerutti T, et al. Elevated level of cell-free plasma DNA is associated with advanced-stage breast cancer and metastasis. Clin Chem Lab Med. 2013;51(11):e277-e278.

68. Chimonidou M, Kallergi G, Georgoulias V, Welch DR, Lianidou ES. BRSM1 promoter methylation provides prognostic information in primary breast tumors. Mol Cancer Res. 2013;11(10):1248-1257.

69. Cáceres IDC, Valmas N, Hilliard MA, Lu H. Laterally orienting $C$. elegans using geometry at microscale for high-throughput visual screens in neurodegeneration and neuronal development studies. PLoS One. 2012;7(4): e35037.

70. deVos T, Tetzner R, Model F, et al. Circulating methylated SEPT9 DNA in plasma is a biomarker for colorectal cancer. Clin Chem. 2009; 55(7):1337-1346.

71. Nakamura J, Tanaka T, Kitajima Y, Noshiro H, Miyazaki K. Methylation-mediated gene silencing as biomarkers of gastric cancer: a review. World J Gastroenterol. 2014;20(34):11991-12006.

72. Kuniholm MH, Lesi OA, Mendy M, et al. Aflatoxin exposure and viral hepatitis in the etiology of liver cirrhosis in the Gambia, West Africa. Environ Health Perspect. 2008;116(11):1553-1557.

73. Schwarzenbach H, Hoon DS, Pantel K. Cell-free nucleic acids as biomarkers in cancer patients. Nat Rev Cancer. 2011;11(6):426-437.

74. Aravanis AM, Lee M, Klausner RD. Next-generation sequencing of circulating tumor DNA for early cancer detection. Cell. 2017;168(4): 571-574.

75. Herrera LJ, Raja S, Gooding WE, et al. Quantitative analysis of circulating plasma DNA as a tumor marker in thoracic malignancies. Clin Chem. 2005;51(1):113-118.

76. Sozzi G, Conte D, Mariani L, et al. Analysis of circulating tumor DNA in plasma at diagnosis and during follow-up of lung cancer patients. Cancer Res. 2001;61(12):4675-4678.

77. Iqbal S, Vishnubhatla S, Raina V, et al. Circulating cell-free DNA and its integrity as a prognostic marker for breast cancer. Springerplus. 2015;4:265.

78. Fujita N, Nakayama T, Yamamoto N, et al. Methylated DNA and total DNA in serum detected by one-step methylation-specific PCR is predictive of poor prognosis for breast cancer patients. Oncology. 2012;83(5): 273-282.

79. Gifford G, Paul J, Vasey PA, Kaye SB, Brown R. The acquisition of hMLH1 methylation in plasma DNA after chemotherapy predicts poor survival for ovarian cancer patients. Clin Cancer Res. 2004;10(13): 4420-4426.

80. Romanel A, Gasi Tandefelt D, Conteduca V, et al. Plasma AR and abiraterone-resistant prostate cancer. Sci Transl Med. 2015;7(312): 312re 10 .

81. Gray ES, Rizos H, Reid AL, et al. Circulating tumor DNA to monitor treatment response and detect acquired resistance in patients with metastatic melanoma. Oncotarget. 2015;6(39):42008-42018.

82. Thierry AR, Mouliere F, El Messaoudi S, et al. Clinical validation of the detection of KRAS and BRAF mutations from circulating tumor DNA. Nat Med. 2014;20(4):430-435.

83. Alegre E, Fusco JP, Restituto P, et al. Total and mutated EGFR quantification in cell-free DNA from non-small cell lung cancer patients detects tumor heterogeneity and presents prognostic value. Tumour Biol.2016; 37(10):13687-13694.
84. Garzón M, Villatoro S, Teixidó C, et al. KRAS mutations in the circulating free DNA (cfDNA) of non-small cell lung cancer (NSCLC) patients. Transl Lung Cancer Res. 2016;5(5):511-516.

85. Dawson SJ, Tsui DW, Murtaza M, et al. Analysis of circulating tumor DNA to monitor metastatic breast cancer. NEngl J Med. 2013; 368(13):1199-1209.

86. Mok T, Wu YL, Lee JS, et al. Detection and dynamic changes of EGFR mutations from circulating tumor DNA as a predictor of survival outcomes in NSCLC patients treated with first-line intercalated erlotinib and chemotherapy. Clin Cancer Res. 2015;21(14):3196-3203.

87. Nygaard AD, Holdgaard PC, Spindler KL, Pallisgaard N, Jakobsen A. The correlation between cell-free DNA and tumour burden was estimated by PET/CT in patients with advanced NSCLC. Br J Cancer. 2014;110(2):363-368.

88. Lipson EJ, Velculescu VE, Pritchard TS, et al. Circulating tumor DNA analysis as a real-time method for monitoring tumor burden in melanoma patients undergoing treatment with immune checkpoint blockade. J Immunother Cancer. 2014;2(1):42.

89. Tie J, Kinde I, Wang Y, et al. Circulating tumor DNA as an early marker of therapeutic response in patients with metastatic colorectal cancer. Ann Oncol. 2015;26(8):1715-1722.

90. Guo N, Lou F, Ma Y, et al. Circulating tumor DNA detection in lung cancer patients before and after surgery. Sci Rep. 2016;6:33519.

91. Bidard FC, Madic J, Mariani P, et al. Detection rate and prognostic value of circulating tumor cells and circulating tumor DNA in metastatic uveal melanoma. Int J Cancer. 2014;134(5):1207-1213.

92. Dawson SJ, Rosenfeld N, Caldas C. Analysis of circulating tumor DNA to monitor metastatic breast cancer. $N$ Engl J Med. 2013; 368(13):1199-1209.

93. Francis G, Stein S. Circulating cell-free tumour DNA in the management of cancer. Int J Mol Sci. 2015;16(6):14122-14142.

94. Ryan BM, Lefort F, McManus R, et al. A prospective study of circulating mutant KRAS2 in the serum of patients with colorectal neoplasia: strong prognostic indicator in postoperative follow up. Gut. 2003;52(1): 101-108.

95. Frattini M, Gallino G, Signoroni S, et al. Quantitative and qualitative characterization of plasma DNA identifies primary and recurrent colorectal cancer. Cancer Lett. 2008;263(2):170-181.

96. Lecomte T, Berger A, Zinzindohoué F, et al. Detection of free-circulating tumor-associated DNA in plasma of colorectal cancer patients and its association with prognosis. Int $J$ Cancer. 2002;100(5):542-548.

97. Olsson E, Winter C, George A, et al. Serial monitoring of circulating tumor DNA in patients with primary breast cancer for detection of occult metastatic disease. Embo Mol Med. 2015;7(8): 1034-1047.

98. Qian X, Liu J, Sun Y, et al. Circulating cell-free DNA has a high degree of specificity to detect exon 19 deletions and the single-point substitution mutation L858R in non-small cell lung cancer. Oncotarget. 2016;7(20):29154-29165.

99. Yanagita M, Redig AJ, Paweletz CP, et al. A prospective evaluation of circulating tumor cells and cell-free DNA in EGFR-mutant non-small cell lung cancer patients treated with erlotinib on a Phase II trial. Clin Cancer Res. 2016;22(24):6010-6020.

100. Tabernero J, Lenz HJ, Siena S, et al. Analysis of circulating DNA and protein biomarkers to predict the clinical activity of regorafenib and assess prognosis in patients with metastatic colorectal cancer: a retrospective, exploratory analysis of the CORRECT trial. Lancet Oncol. 2015;16(8):937-948.

101. Namløs HM, Zaikova O, Bjerkehagen B, et al. Use of liquid biopsies to monitor disease progression in a sarcoma patient: a case report. BMC Cancer. 2017;17(1):29.

102. Casadevall D, Vidal J, Gallardo F, et al. Dabrafenib in an elderly patient with metastatic melanoma and BRAF V600R mutation: a case report. J Med Case Rep. 2016;10(1):158.

103. Carpinetti P, Donnard E, Bettoni F, et al. The use of personalized biomarkers and liquid biopsies to monitor treatment response and disease recurrence in locally advanced rectal cancer after neoadjuvant chemoradiation. Oncotarget. 2015;6(35):38360-38371. 
104. Chedgy EC, Annala M, Beja K, et al. Moving toward personalized care: liquid biopsy predicts response to cisplatin in an unusual case of BRCA2-null neuroendocrine prostate cancer. Clin Genitourin Cancer. 2016;14(2):e233-e236.

105. Nilbert M, Meza-Zepeda LA, Francis P, et al. Lessons from genetic profiling in soft tissue sarcomas. Acta Orthop Scand Suppl. 2004; 75(311):35-50.

106. Lopes-Ramos C, Koyama FC, Habr-Gama A, et al. Comprehensive evaluation of the effectiveness of gene expression signatures to predict complete response to neoadjuvant chemoradiotherapy and guide surgical intervention in rectal cancer. Cancer Genet. 2015;208(6):319-326.
107. Nakagomi H, Hirotsu Y, Amemiya K, et al. Rapid changes in circulating tumor DNA in serially sampled plasma during treatment of breast cancer: a case report. Am J Case Rep. 2017;18:26-32.

108. Schwaederle M, Husain H, Fanta PT, et al. Use of liquid biopsies in clinical oncology: pilot experience in 168 patients. Clin Cancer Res. 2016;22(22):5497-5505.

\section{Publish your work in this journal}

Therapeutics and Clinical Risk Management is an international, peerreviewed journal of clinical therapeutics and risk management, focusing on concise rapid reporting of clinical studies in all therapeutic areas, outcomes, safety, and programs for the effective, safe, and sustained use of medicines. This journal is indexed on PubMed Central, CAS,
EMBase, Scopus and the Elsevier Bibliographic databases. The manuscript management system is completely online and includes a very quick and fair peer-review system, which is all easy to use. Visit http://www.dovepress.com/testimonials.php to read real quotes from published authors.

Submit your manuscript here: http://www.dovepress.com/therapeutics-and-clinical-risk-management-journal 